\title{
MODELO DE NEGOCIACIÓN DE RESIDUOS SÓLIDOS ORGÁNICOS EN CIUDAD BOLIVAR, BOGOTÁ
}

\section{Business organic solid waste model in Ciudad Bolívar, Bogotá}

YILMER GIRALDO RODRÍGUEZ CAMPOS

yilmer.rodriguez@uniminuto.edu.co

ERIKA MARCELA TRUJILLO CRUZ

erika.trujillo@uniminuto.edu.co,

YESSICA PAOLA MORALES MADRID

yessica.morales@uniminuto.edu.co

JENSY CAMILA MONTIEL CORTES

jensy.montiel@uniminuto.edu.co

LUIS ALEJANDRO NÚÑEZ ROMERO

luis.nunez-r@uniminuto.edu.co

LEONOR URIBE VARGAS

leonor.uribe@uniminuto.edu.co

JEFFER ALEJANDRO PEÑA PINILLA

jeffer.pena@uniminuto.edu.co

JENNY ALEXANDRA SEGURA OSUNA (Docente investigador) jseguraousn@uniminuto.edu.co

Corporación Universitaria Minuto de Dios (Colombia)

Artículo de investigación formativa

Recepción: 30 de noviembre de 2020

Aceptación: 5 de marzo de 2021 


\section{Resumen:}

La investigación del semillero está enfocada en la responsabilidad social y el impacto que genera al medio ambiente la degradación de los desechos orgánicos que a diario son depositados en los rellenos sanitarios en Bogotá. Como principal hallazgo, la actividad del reciclaje proviene de tradición familiar por falta de oportunidades para la obtención de ingresos. Entonces, se evidencia la importancia de realizar un trato especial a los desechos y vincular en esta labor a diferentes entes como restaurantes y plazas de mercado; ellos originan gran parte de estos residuos. Así, se considera un enfoque de investigación mixto (documental y acción participativa). El principal objetivo busca en cuatro (4) fases plantear un modelo de negociación entre compradores y vendedores de reciclaje de residuos sólidos orgánicos que dependen económicamente de su recolección. Además, para mostrar la correcta disposición de los residuos sólidos orgánicos a emplear en diferentes productos que benefician a las familias, está enfocado en la localidad de Ciudad Bolívar, Bogotá.

Palabras clave: residuos orgánicos; medio ambiente; plan de negocio; relleno sanitario; desechos.

\section{Abstract:}

The investigation is focus on social responsibility and the impact that the degradation of the organic rights (diary deposited in landfills in Bogotá) generates to the environment means. As the principal finding, the recycle activity comes from a lack of opportunities for obtaining incomes, due to a familiar tradition. In this way, it evidences the importance to make an especial treatment with the waste and link up this labor to different entities as restaurants and marketplaces; they originate big part of the wastes. It considers a mix investigation type (documental and participative action). The main objective seeks to pose a business model between sellers and buyers of solid organic wastes recycle that economically depends on its recollection; all in four (4) phases. What's more, to show the correct disposition of the organic solids wastes to use in different products that benefit families and is focuses in Ciudad Bolivar, town in Bogotá, Colombia.

Keywords: organic wastes; environment; business plan; landfill; wastes. 


\section{Introducción}

Según Rodríguez y Chávez (2016), en el 2012 del total de residuos sólidos (RS), el 46\% corresponde a residuos orgánicos (RO). Las 3R: reducir, reciclar y reutilizar como método para fomentar formas de producción más "limpias" y sostenibles en el largo plazo. Hay países en los cuales se han desarrollado estrategias para el aprovechamiento de los RS, como materia prima para producir otros bienes.

Sin embargo, hay carencia en cuanto al número de programas que se dedican a eso, dado que no tienen el apoyo suficiente, especialmente porque falta en los ciudadanos un cambio de mentalidad y comportamiento frente al tratamiento que se le da a los RS. A diferencia de países como España, Colombia no ha formulado programas y políticas lo cual hace que en la actualidad haya muchos rellenos sanitarios copados con RS, que dejan una huella ambiental negativa.

La localidad número 19 de Ciudad Bolívar en la ciudad de Bogotá se encuentra ubicada al sur de la ciudad; colinda al norte con la localidad de Bosa, al occidente con el municipio de Soacha, al oriente con las localidades de Tunjuelito y Usme; por último, al sur con las localidades de Usme y Sumapaz. Su ocupación a partir de asentamientos de parcelas data de los años 1940, desde los cuales ha venido creciendo, en medio de zona montañosas y zonas rurales. Estás ultimas ocupan el $72 \%$ del total de la localidad (Alcaldía Local de Ciudad Bolívar, s.f; Rodríguez-Parra y Acosta-Triviño, 2017)

A dicha localidad llega al relleno sanitario de Doña Juana un tipo de RS, son lo que provienen de material orgánico como plantas o animales y generalmente se pueden biodegradar fácil y rápidamente transformándose en otro tipo de materia orgánica (Jaramillo y Zapata, 2008). Ejemplo de ello son los restos vegetales de animales (carne, huesos, piel), asimismo son putrefactos, sin embargo, pueden reutilizarse y aprovecharse.

Además, en Colombia se ha avanzado hacia el aprovechamiento de RS, tanto de tipo orgánico como inorgánico. No obstante, no hay modelos de negociación que hayan sido propuestos y utilizados por aquellos agentes que intervienen en las negociaciones de compra y venta en dichos residuos. Lo anterior se refleja en el hecho que en la actualidad la mayoría de los RS orgánicos van a parar a los rellenos sanitarios municipales. En el caso de Bogotá el principal es el relleno sanitario Doña Juana, ubicado en el sur de la ciudad desde el primero de noviembre de 1988, cuando comenzó operaciones según lo indica la Unidad Administrativa Especial de Servicios Públicos (UAESP, 2020).

\section{Metodología}

Este semillero quiere mostrar cómo los residuos orgánicos se pueden reciclar de manera responsable para ser reutilizados y no solo eliminarlos en bolsas de basura con otro tipo de residuos que van a llegar al relleno de Doña 
Juana. Alli se descomponen y crean un gran problema para aquellos que viven cerca; los afecta desde diferentes maneras (salud, roedores, contaminación ambiental, entre otros) y para toda la ciudad de Bogotá.

De esta manera, la pregunta problema es: ¿Cómo vincular a los recicladores en el proceso de reciclaje de residuos orgánicos en Bogotá? Durante la investigación adelantada hasta el momento, se evidencia que en Bogotá son muchas las familias que dependen económicamente de su labor de reciclaje. Además, como muchas de ellas no se encuentran vinculadas a ningún programa del Gobierno, tampoco a algún centro de reciclaje, trabajan por su cuenta en lo que a diario puedan conseguir para venderlo y obtener un beneficio económico. Con base en esta información se formula el objetivo general, así como algunos objetivos específicos, a continuación.

\section{Objetivos}

\section{General.}

Generar un modelo donde se vincule a los recicladores para trabajar con los residuos sólidos orgánicos y no solos con residuos sólidos inorgánicos.

\section{Específicos.}

- Realizar encuestas con el fin de poder obtener información de primera mano. - Generar un modelo de gestión de residuos orgánicos que beneficie a los recicladores de Bogotá.

- Identificar oportunidades a desarrollar para la correcta recolección de los residuos y su transporte.

Los residuos sólidos orgánicos se pueden transformar en diferentes formas como el compostaje, los biocombustibles, objetos de joyería, comida para animales entre otros. Por consiguiente, se podría llevar a cabo las 3R como método para fomentar formas de producción más "limpios" y sostenibles en el largo plazo.

Es conocido que los residuos sólidos orgánicos se generan principalmente en plazas de mercado, restaurantes, fruterías y por supuesto en los hogares. Desafortunadamente en Colombia no se tiene una educación en la separación de los residuos; son mal dispuestos en bolsas de basura que en su mayoría llegan al botadero de Doña Juana donde la descomposición genera un gran foco de contaminación y problemas de salud a los habitantes que viven en cercanías del botadero al sur de Bogotá. Por tanto, la investigación se hace en cuatro (4) fases:

1.Revisión literaria.

2.Construcción de herramientas de trabajo.

3.Encuestas y entrevistas o compradores y vendedores.

4.Análisis de resultados. 
Durante la investigación se han realizado encuestas a los recicladores y también a pequeños mercados (fruvers) y fruterías con el fin de recaudar la mayor información de primera mano de los entes implicados en el proceso del manejo de los residuos sólidos orgánicos en Bogotá. Una vez resuelto lo anterior, se elabora la fase cuatro: el análisis de los resultados obtenidos en las encuestas desarrolladas.

\section{Discusión y resultados}

Con la presente investigación hasta el momento se ha encontrado que la actividad del reciclaje proviene de tradición familiar por falta de oportunidades para la obtención de ingresos. Algunos logran organizarse mediante cooperativas, asociaciones y emprendimiento, pero su gran mayoría lo hace de manera informal, como medio para poder subsistir y sacar adelante a sus familias, poder brindarles lo mejor posible en medio de sus necesidades.

En efecto, se plantea la necesidad de inclusión sostenible de los recicladores informales al esquema de servicio de aseo público de Bogotá, evidenciando la no oferta de condiciones laborales óptimas para el desarrollo de dicha actividad. Asimismo, se ha evidenciado que al utilizar los Residuos Sólidos Orgánicos (RSO) como fuente de aprovechamiento para la obtención de otros bienes que se pueden conseguir diferentes al compost (el más conocido y aplicado) como el biocombustible, elementos para nanotecnologías, colchones, ladrillos, papel, entre otros.

En este sentido, permite la descontaminación del medio ambiente y la conservación de la salud de las personas, especialmente de aquellas que se ubican en las zonas aledañas al relleno sanitario de Doña Juana.

\section{Conclusiones}

Trabajar en conjunto con la comunidad es satisfactorio porque permite contribuir en el proceso de reciclaje que manejan, en mejorar los métodos para una mejor calidad de vida. Esta investigación busca soluciones al problema que se presenta en el sector y en la sociedad en general, evitando el desperdicio de la materia prima y los recursos no renovables, ya que es muy importante la preservación del medio ambiente.

Durante la investigación se evidencian las necesidades de todas aquellas personas que viven del reciclaje. Muchos de ellos manifiestan que durante años y, en algunos durante toda su vida, esta ha sido su única fuente de ingresos. Lo que para la mayoría es basura, para ellos es una oportunidad de trabajo que les ha permitido sacar adelante a sus familias de una forma digna. Compartir con la comunidad genera satisfacción y brindar información que pueda ayudarles y darles nuevas alternativas de trabajo es gratificante. De una u otra forma se está contribuyendo a mejorar su calidad de vida. 


\section{Referencias bibliográficas}

Alcaldía Local de Ciudad Bolívar. (s.f.). Conociendo mi localidad. Ciudad Bolívar. Recuperado de http://www. ciudadbolivar.gov.co/mi-localidad/ conociendo-mi-localidad/historia

Jaramillo, G. y Zapata, L. M. (2008). Aprovechamiento de los residuos sólidos orgánicos en Colombia (trabajo de especialización). Universidad de Antioquia, Medellín, Colombia. Recuperado de http://bibliotecadigital.udea.edu.co/dspace/ bitstream/10495/45/1/AprovechamientoRSOUenColombia.pdf

Rodríguez, A. y Chávez, Á. (2016). Aprovechamiento de residuos orgánicos agrícolas y forestales en Iberoamérica. Academia y virtualidad, 9(2), 90-107. DOl https://doi. org/10.18359/ravi.2004

Rodríguez-Parra, P. y Acosta-Triviño, R. (2017). Delitos y conflicto en la localidad 16 Puente Aranda. Bogotá: Fundación Universitaria San Mateo.

UAESP. (2020). Especiales UAESP Relleno Sanitario Doña Juana. UAESP. Recuperado de: http:// www.uaesp.gov.co/especiales/relleno/ 